\section{Research Article}

J Ginseng Res Vol. 37, No. 4, 425-434 (2013)

http://dx.doi.org/10.5142/jgr.2013.37.425

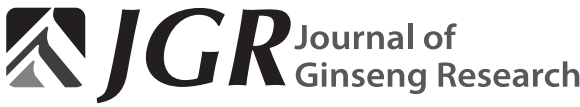

\title{
Enzyme-processed Korean Red Ginseng extracts protects against skin damage induced by UVB irradiation in hairless mice
}

\author{
Eunson Hwang ${ }^{1}$, Zheng-wang Sun ${ }^{1}$, Taek Hwan Lee ${ }^{2}$, Heon-Sub Shin ${ }^{1}$, Sang-Yong Park ${ }^{1}$, Don-Gil Lee ${ }^{1}$, \\ Byung-Goo Cho ${ }^{3}$, Hyunjoo Sohn ${ }^{4}$, Oh Wook Kwon ${ }^{5}$, Sun Yeou Kim, ${ }^{6,7^{*}}$, and Tae Hoo $\mathrm{Yi}^{1^{*}}$ \\ ${ }^{1}$ Department of Oriental Medicinal Material and Processing, College of Life Science, Kyung Hee University, Yongin 446-701, \\ Korea \\ ${ }^{2}$ College of pharmacy, Yonsei University, Incheon 460-840, Korea \\ ${ }^{3} \mathrm{R} \& D$ Headquarters, Korea Ginseng Corporation, Daejeon 305-805, Korea \\ ${ }^{4}$ Korea Ginseng Research Institute, Daejeon 305-805, Korea \\ ${ }^{5}$ Graduate School of East-West Medical Science, Kyung Hee University, Yongin 446-701, Korea \\ ${ }^{6}$ Gachon Institute of Pharmaceutical Science, Gachon University, Incheon 406-799, Korea \\ ${ }^{7}$ College of Pharmacy, Gachon University, Incheon 406-799, Korea
}

UV irradiation is the main factor contributing to skin damages that are associated with an excessive production of matrixdegrading metalloproteinase (MMP)-1 and a deficient expression of collagens. To date, red ginseng has been revealed to possess many biomedical effects, such as anti-aging, anti-oxidation, and anti-inflammatory. In this study, we prepared the Korean Red Ginseng extracts treated with enzyme (KRGE) and investigated the effects of dietary KRGE on the formation of wrinkles generated by UVB irradiation in hairless mice. It was found that KRGE inhibited the UVB-induced formation of wrinkles, epidermal thickness, and skin dryness in hairless mice. Further results also showed that KRGE attenuated UVB-induced MMP-1 level, while accelerated procollagen type I, transforming growth factor- $\beta 1$ secretion. Interestingly, the expression of profilaggrin and filaggrin in both the epidermis and dermis were decreased due to UVB exposure and reversed by KRGE. The KRGE 0.06\% was prior to KRGE 0.24\%. In view of these results, which indicated that KRGE protected skin from UVB-induced photodamages, which may not only mediated by regulating of MMP-1 and procollagen type I, but also by increasing the production of profilaggrin and filaggrin. In conclusion, our results suggest that KRGE may be a promising agent for the treatment of skin photodamages. The challenge of KRGE will be expected as cosmeceuticals and nutraceuticals in order to intervene in aging-related degenerative skin changes.

Keywords: Panax ginseng, Red ginseng, Enzyme, Photoaging, Skin hydration

\section{INTRODUCTION}

Aging is a biological process that changes structural integrity and physiological function [1]. Skin exposure to UV radiation, particularly its UVB component (280 to $320 \mathrm{~nm}$ ), results in photoaging characterized by pigmentation, roughness, dryness, wrinkle formation, variable

cc This is an Open Access article distributed under the terms of the Creative Commons Attribution Non-Commercial License (http://creativecommons.org/licenses/by-nc/3.0/) which permits unrestricted non-commercial use, distribution, and reproduction in any medium, provided the original work is properly cited.

(C) The Korean Society of Ginseng epidermal thickness, and disruption of the epidermal barrier [2]. Photoaging is triggered by reactive oxygen species as well as the transcription factor activator protein (AP-1) [3]. Increased expression of AP-1 activates the transcription of matrix-degrading metalloproteinases

Edited by Jong-Hoon Kim, Chonbuk National University, Korea

Received 26 Feb. 2013, Revised 04 Jul. 2013, Accepted 04 Jul. 2013

*Corresponding authors

E-mail: sunnykim@gachon.ac.kr

Tel: +82-32-899-6411, Fax: +82-32-899-6591

E-mail: drhoo@khu.ac.kr

Tel: +82-31-201-3693, Fax: +82-31-206-3693 
(MMPs), which are a zinc-dependent endoproteases responsible for collagen degradation [4]. In particular, MMP-1 inhibits the synthesis of major dermal collagens by blocking the activity of transforming growth factor (TGF)- $\beta$, which in turn enhances transcription of collagen genes $[5,6]$. Dermal fibroblasts form precursor molecules called procollagen, which are converted into collagen. Further, collagen fragments negatively regulate the synthesis of new collagen [7]. Therefore, due to the process of constant degradation of procollagen mediated by MMPs, collagen production is reduced in photoaged skin.

UVB radiation alters epidermal morphology by increasing and unbalancing stratum corneum (SC) thickness [8], and such disorganization of the SC permeability barrier can increase trans-epidermal water loss [9]. Several studies have investigated the histological and functional responses of the epidermis to UV irradiation [7,10], including loss of water-holding properties [9]. Filaggrin and profilaggrin are essential for maintaining homeostasis in the epidermis. Filaggrin also undergoes further processing in the upper SC to release free amino acids that assist in water retention [11]. However, little is known about the relationship between wrinkle formation and epidermal hydration related proteins.

Panax ginseng Meyer has been widely used as a traditional oriental medicine for various diseases, including atherosclerosis, liver dysfunction, cerebrovascular disease, hypertension, and post-menopausal disorder [12]. The main pharmacologically active components of ginseng are believed to be ginsenosides, which are derivatives of triterpene dammaranes [13] and possess antioxidant, antistress, anticancer and pro-immune properties [14-17]. Commonly available ginseng is divided into fresh, white, and red ginseng. White ginseng is a powerful herb that stimulates the body's systems and helps to reduce stress, boost energy and sustain body fuctions [18]. Red ginseng is prepared by steaming and drying and can be kept for long periods of time without loss of its biological activities. Red ginseng extracts have also been shown to be effective for treating atopic suppurative dermatitis, wounds, and skin inflammation [19-21]. This research has focused mostly on dermatological application of ginseng in cosmetics and medicines. On the other hand, our research is partially conducted in vivo for the purpose of exploring its application in functional foods. Furthermore, the protective effects of oral administration of Korean Red Ginseng extract treated with enzyme (KRGE) against skin photoaging from UVB irradiation have not been reported. In this study, we investigated whether KRGE prevents UVB irradiation-induced photodamage in aging hairless mice skin.

\section{MATERIALS AND METHODS}

\section{Materials}

Six-year-old Korean Red Ginseng (KRG) was provided by Dr. Byung-Goo Cho (R\&D Headquarters, Korea Ginseng Corporation, Daejeon, Korea). Standard ginsenosides, including the compounds Rg1, Re, Rf, Rh1, Rg2, Rb1, Rb2, Rc, Rd, Rg3, Rh2, and F2 were purchased from Ambo Laboratory (Daejeon, Korea). All other chemicals were of analytical grade and obtained from local suppliers.

\section{Preparation of Korean Red Ginseng extract treated with enzyme}

KRGE was obtained with its patented protocol (Korea patent no. 10-2011-0091287 [in private], in press). Briefly, the crude enzyme was extracted from Aspergillus niger (KACC 40280) which was isolated from the Nuruk for Korean traditional wine. Then the active enzyme was purified by loading into an ion-exchage resin. The dried and grinded KRG $(0.5 \mathrm{~kg})$ was incubated with the enzyme solution containing ginsenoside- $\beta$-glucosidase and extracted from 5.0 L of ethanol. KRG had reacted with the enzyme at $50^{\circ} \mathrm{C}$ to $60^{\circ} \mathrm{C}$ for $24 \mathrm{~h}$. After reaction, the enzyme was removed by ultrafiltration (MWCO, 10,000 $\mathrm{Da})$. Then the solution was filtered and concentrated.

\section{Ultra performance liquid chromatography-photo- diode array analysis}

Ultra performance liquid chromatography (UPLC) was performed on a Waters ACQUITY UPLC system (Waters, Mildford, MA, USA) equipped with a binary solvent delivery system, an autosampler and a UV detector. Chromatographic separation was performed on a Waters Acquity HPLC BEH C ${ }_{18}$ column $(50 \times 2.1 \mathrm{~mm}$ internal diameter, $1.7 \mu \mathrm{m}$ particle size). The elution was performed by an acetonitrile/water gradient containing $0.1 \%$ formic acid. Runs were carried out in isocratic mode at a flow rate of $0.6 \mathrm{~mL} / \mathrm{min}$. Total run time, including re-equilibration of the column to the initial conditions, was $27 \mathrm{~min}$. Samples of $2 \mu \mathrm{L}$ were injected into the UPLC system. The detection range of the photodiode array detector was set between 190 and $500 \mathrm{~nm}$.

\section{Animals}

Seven-week-old male albino hairless mice (SKH: HR-1) (20-27 g; $n=40)$ were obtained from Central Lab 
Animals (Seoul, Korea). The experimental protocol for this study (KHUASP(SU)-12-09) was approved by the Institutional Animal Care and Use Committee of Kyung Hee University. Mice were housed in a temperature- and humidity-controlled room $\left(22 \pm 1^{\circ} \mathrm{C}, 60 \pm 5 \%\right.$ humidity $)$ with $12 \mathrm{~h}$ light/ dark cycles. After $1 \mathrm{wk}$ of quarantine, mice were acclimated to individual cages.

\section{UV irradiation}

The UV source was supplied by a closely spaced array of five Sankyo Denki sunlamps, which have a peak irradiance at $310 \mathrm{~nm}$ (G9T5E lamps; Sankyo Denki, Hiratsuka, Japan). Bulbs were positioned $15 \mathrm{~cm}$ above the mice. Irradiance $\left(0.1 \mathrm{~mW} / \mathrm{cm}^{2}\right)$ was measured with an IL1700 Research Radiometer (International Light, Newburyport, MA, USA) equipped with a UVB sensor. Mice were exposed to $100 \mathrm{~mJ} / \mathrm{cm}^{2}$ UVB radiation (1 minimal erythematal dose $=100 \mathrm{~mJ} / \mathrm{cm}^{2}$ ) seven times per week for the first week, and thereafter at $200 \mathrm{~mJ} / \mathrm{cm}^{2}$ twice a week for 9 wk. UVB irradiation was applied to the dorsal skin of mice and no injury to the dorsal skin was observed with the doses of UVB irradiation administered in this study.

\section{Diet}

We randomly divided 40 hairless mice into four groups of ten mice per cage: 1) normal (control diet only), 2) control (UVB irradiation + control diet), 3) KRGE 0.06\% (UVB irradiation + diet containing 0.06\% KRGE), and 4) KRGE $0.24 \%$ (UVB irradiation + diet containing $0.24 \%$ KRGE). All of the mice were fed solid formula feed. During the experimental period, access to food and water was provided ad libitum. Animals in each group received the experimental diets for $10 \mathrm{wk}$ concurrent with the UVB irradiation regimen. The compositions of the experimental diets given to each group are shown in Table 1. The amount of dietary fat (supplied as corn oil) was fixed as $10 \%$ of the dietary weight. Food intake and body weight were measured once a week for all groups.

\section{Wrinkle measurement}

To obtain skin replicas, $1.5 \mathrm{~g}$ of light-bodied silicone (SilfloR; Flexico, Colchester, UK) was prepared from two components mixed at a ratio of 1:1 (one drop each of catalyst and thinner). The mixture was then applied to the skin surface. After drying and hardening, replicas were subjected to further analysis. The visiometer technique was used to detect changes in the transparency of thin silicone gel prints of skin surfaces and collected with a charge-coupled device video camera. Under our condi-
Table 1. Composition of diet ( $\mathrm{g} / \mathrm{kg}$ dry diet)

\begin{tabular}{lcccc}
\hline & \multicolumn{4}{c}{ Experimental groups } \\
\cline { 2 - 5 } & $\begin{array}{c}\text { Normal } \\
(n=10)\end{array}$ & $\begin{array}{c}\text { UVBcont } \\
(n=10)\end{array}$ & $\begin{array}{c}\text { KRGE 0.06\% } \\
(n=10)\end{array}$ & $\begin{array}{c}\text { KRGE 0.24\% } \\
(n=10)\end{array}$ \\
\hline Casein & 230 & 230 & 230 & 230 \\
L-cystine & 3 & 3 & 3 & 3 \\
Corn oil & 100 & 100 & 100 & 100 \\
Cellulose & 50 & 50 & 50 & 50 \\
Vitamin mix & 10 & 10 & 10 & 10 \\
Mineral mix & 35 & 35 & 35 & 35 \\
Sucrose & 200 & 200 & 200 & 200 \\
Corn starch & 372 & 372 & 371.4 & 369.6 \\
KRGE & - & - & 0.6 & 2.4 \\
UVB irradiation & $\times$ & $\circ$ & $\circ$ & $\circ$ \\
\hline
\end{tabular}

Group normal, control diet only; group UVBcont, UVB irradiation + control diet; group Korean Red Ginseng extract treated with enzyme (KRGE) 0.06\%, UVB irradiation + diet containing 0.06\% KRGE; group KRGE $0.24 \%$, UVB irradiation + diet containing $0.24 \% \mathrm{KRGE}$; $\times$ means non-UV irradiated hairless mice and $\circ$ means UV irradiated hairless mice.

tions, the angle of incidence of light was fixed at $45^{\circ} \mathrm{C}$ relative to the skin replica surface, and the resulting black and white images were recorded to produce an image file. The image files were analyzed using Skin Viscometer SV 600 software (Courage \& Khazaka, Cologne, Germany). Arbitrary units (R1 to R5) were assigned to each sample based on measurements of the depth of furrows according to shadow size and brightness due to inflection under illumination. Skin roughness (R1) was defined as the difference between the highest crest and lowest furrow. To exclude the possibility of artifacts, the program cut each line into five equal parts, with R3 representing an average of the maximum distance (R1) derived from each of the five parts of the line. Likewise, R2 represented the largest value of the five distances while R4 represented the mean area surrounded by a horizontal line drawn at the highest crest and the furrows profile. R5 was taken as the mean deviation of the furrow's profile to the middle line.

\section{Measurement of physiological skin functions}

During the study, erythema values and water content of the SC were measured after feeding and irradiation with an analytical system and appropriate probes (DermaLab Combo; Cortex Technology, Hadsund, Denmark). Erythema values and SC hydration levels in the dorsal skin surface of the hairless mice were measured as described above. 


\section{Histological analysis}

Mice were sacrificed after the final UVB exposure and biopsies were obtained from the dorsal skin. Biopsies were fixed in $4 \%$ paraformaldehyde for $24 \mathrm{~h}$ and embedded in paraffin. Sections approximately $4 \mu \mathrm{m}$ thick were stained with hematoxylin for $10 \mathrm{~min}$, washed, then stained with eosin for $2 \mathrm{~min}$. After washing with water, the slides were gradually dehydrated in $50 \%, 70 \%, 90 \%$, and $100 \%$ ethanol. Other samples were stained with Masson's trichrome to examine collagen density in the dermis. These sections were first stained with Bouin solution at $56^{\circ} \mathrm{C}$ for $1 \mathrm{~h}$, followed by washing and then staining with Weigert's iron hematoxylin working solution for $10 \mathrm{~min}$. Slides were subsequently washed and differentiated in phosphomolybdic-phosphotungstic acid solution for 10 to $15 \mathrm{~min}$, then stained with an aniline blue solution for 5 to $10 \mathrm{~min}$. After washing, the slides were quickly dehydrated using sequential washes with $95 \%$ and $100 \%$ ethanol. Stained slides were examined with a light microscope.

\section{Western blot analysis}

Western blotting was performed using total cell and tissue lysates. Following treatment, cells were harvested and washed in phosphate buffered saline. Cell and tissue samples were homogenized with lysis buffer containing $50 \mathrm{mM}$ Tris-Cl, $\mathrm{pH}$ 8.0, 0.1\% sodium dodecyl sulfate (SDS), $150 \mathrm{mM} \mathrm{NaCl}, 1 \%$ Nonidet P-40, 0.02\% sodium azide, $0.5 \%$ sodium deoxycholate, $100 \mu \mathrm{g} / \mathrm{mL}$ phenylmethylsulfonyl fluoride, $1 \mu \mathrm{g} / \mathrm{mL}$ aprotinin, and a phosphatase inhibitor. The lysates were then subjected to centrifugation at 12,000 $\mathrm{g}$ for $20 \mathrm{~min}$. All cell and tissue lysates were analyzed in triplicate. Protein concentrations were measured using Bradford reagent (Bio-Rad, Hercules, CA, USA) with bovine serum albumin as the standard. Skin tissue lysates containing equal amounts of total protein were separated by $8 \%$ or $10 \%$ SDS-PAGE and then transferred to a nitrocellulose membrane (Amersham Pharmacia Biotech, Buckinghamshire, UK). Next, membranes were blocked with a solution containing 5\% non-fat milk in tris-buffered saline with tween 20 (TBST) for $1 \mathrm{~h}$ at room temperature and then incubated overnight with a primary antibody at $4^{\circ} \mathrm{C}$. The membranes were then washed three times with TBST and incubated with secondary antibodies (Santa Cruz Biotechnology, Santa Cruz, CA, USA) for $1 \mathrm{~h}$ at room temperature. Finally, immune complexes were detected with an ECL Western blot detection system (LAS-4000; Fujifilm, Tokyo, Japan). Densitometry analysis of bands was performed with ImageMaster 2D Elite software, version 3.1 (Amersham Pharmacia Biotech, Piscataway, NJ, USA).
Table 2. Ginsenoside contents of Korean Red Ginseng extract treated with enzyme

\begin{tabular}{llc}
\hline Ginsenosides & Concentration $(\mathrm{mg} / \mathrm{g})$ \\
\hline PPT & Re & 2.05 \\
& Rf & 2.96 \\
& Rh1 & 0.12 \\
& Rg2 & 0.59 \\
& Total & 0.48 \\
PPD & Rb1 & 6.20 \\
& Rb2 & 0.39 \\
& Rc & 0.96 \\
Rd & 0.81 \\
Total & Rg3 & 2.81 \\
& Rh2 & 0.43 \\
& F2 & ND \\
& Total & 3.45 \\
& & 8.85 \\
& & 15.05 \\
& &
\end{tabular}

The ginsenosides of Korean Red Ginseng extract treated with enzyme was analyzed, ultra performance liquid chromatography was accomplished as in materials and methods.

PPT, protopanaxatriol; PPD, protopanaxadiol; ND, non-detectable.

\section{Statistical analysis}

The results were determined using the Statistical Analysis System (GraphPad Prism 5). All experiments were carried out in triplicate. The data are expressed as means \pm standard deviation. Statistical comparisons between different treatment groups were performed using one-way analysis of variance followed by Duncan's test. Student's $t$-test was used to compare individual treatments to controls. The level of statistical significance was set at $p<0.05$.

\section{RESULTS}

\section{Identification of Korean Red Ginseng extract treat- ed with enzyme composition}

As shown in Table 2, the total ginsenoside contents of KRGE was $15.05 \mathrm{mg} / \mathrm{g}$. The protopanaxadiol and protopanaxatriol ginsenosides contained about $6.20 \mathrm{mg} /$ $\mathrm{g}$ and $8.85 \mathrm{mg} / \mathrm{g}$, respectively. Rb1 and Rg1 are the two main ginsenosides concluded in KRGE $(0.39 \mathrm{mg} / \mathrm{g}$ and $2.05 \mathrm{mg} / \mathrm{g}$, respectively). The $\beta$-glycosidase from Aspergillus sp. exploits the hydrolytic pathways. The ginsenoside Rb1, Rb2, or Rg3 transformed into F2, and then $\mathrm{Rh} 2$. Because these $\beta$-d-glycosidases are able to simultaneously hydrolyze, they produce the minor ginsenosides with a high yield, several major ginsenosides [22]. Simi- 


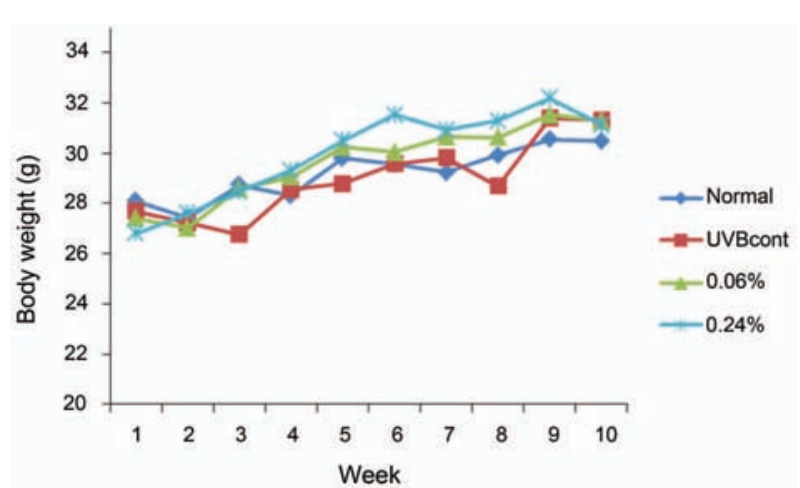

Fig. 1. Weight gain of hairless mice fed control diet only (normal), UVB-irradiated hairless mice fed control diet (UVBcont), UVB-irradiated hairless mice fed containing $0.06 \%$ Korean Red Ginseng extract treated with enzyme (KRGE 0.06\%) and UVB-irradiated hairless mice fed containing $0.24 \%$ KRGE (KRGE 0.24\%) for 10 wk. Values are mean \pm SD $(10 \mathrm{wk}, n=10)$.

Table 3. Food efficiency ratio of experimental groups in $10 \mathrm{wk}$

\begin{tabular}{lcccc}
\hline \multicolumn{4}{c}{ Dietary group } \\
\cline { 2 - 5 } & Normal & UVBcont & KRGE 0.06\% & KRGE 0.24\% \\
FER $^{1)}$ & $36.90 \pm 3.30$ & $33.87 \pm 3.75$ & $35.43 \pm 5.38$ & $37.99 \pm 6.13$ \\
\hline
\end{tabular}

Group normal, control diet only; group UVBcont, UVB irradiation + control diet; group Korean Red Ginseng extract treated with enzyme (KRGE) $0.06 \%$, UVB irradiation + diet containing $0.06 \% \mathrm{KRGE}$; group KRGE 0.24\%, UVB irradiation + diet containing 0.24\% KRGE.

1) FER (food efficiency rate)=gain of body weight $(\mathrm{g}) /$ amount of food intake $(g) \times 100$. Values are mean \pm SD $(n=10)$. Mean with different letters differ $p<0.05$.

larly, our data has been shown that F2 content (3.45 mg/g) showed the highest level in KRGE.

\section{Body weight gain and food efficiency rate}

The average body weight differences among the four groups of mice were not significant after 10 wk. During the 3rd week, the mean body weight of the UVB control group was about $27 \mathrm{~g}$, which was remarkably lower when compared with the normal group, (29 g). However, the body weights of the UVB control and normal groups were similar to each other after $8 \mathrm{wk}$. The average body weights of the KRGE $0.06 \%$ and KRGE $0.24 \%$ groups increased to $31 \mathrm{~g}$ (Fig. 1). The food efficiency rate (body weight [g]/food intake $[\mathrm{g}] \times 100$ ) of the UVB control group was the lowest $(33.87 \pm 3.75)$ among the four groups, while the food efficiency rates of the other groups were not significantly different from that of the control group (normal: 36.90 \pm 3.30 , KRGE 0.06\%:

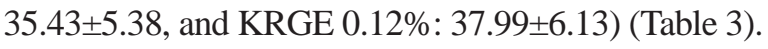

\section{Wrinkle measurement and analysis of skin replicas}

Fig. 2 shows the replicas of the central dorsum of mice. In the non-irradiated group, the skin appeared to be fine with thin wrinkle formation (Fig. 2A). Conversely, deeper and wider wrinkles were observed in the UVBirradiated control group. The KRGE $0.06 \%$ and KRGE $0.24 \%$ groups exhibited reduced UVB-induced wrinkle formation (Fig. 2B). However, no differences were observed between the KRGE $0.06 \%$ and KRGE 0.24\% groups (Fig. 2C).

\section{Inhibition of UVB-induced histological changes following oral administration of Korean Red Gin- seng extract treated with enzyme}

Red ginseng extract has been reported to prevent UVB irradiation-induced skin photoaging [23]. In our study, H\&E staining showed that KRGE inhibited increased epidermis thickness caused by UVB irradiation. Likewise, expansion of skin thickness induced by UVB irradiation appeared to be reversed by KRGE. Compared with the KRGE $0.24 \%$ group, KRGE $0.06 \%$ group was more effective (Fig. 3A).

Masson trichrome staining was used to observe collagen fibers. Compared with the normal group, the UVB control group exhibited decreased abundance and density of collagen fibers. Treatment with KRGE significantly restored the reduction of collagen fibers induced by UVB-irradiation. Furthermore, dietary KRGE at a low dose $(0.06 \%)$ appeared to be more effective at restoring collagen fibers compared with $0.24 \% \mathrm{KRGE}$ (Fig. 3B).

\section{Physiological analysis of mouse skin surface fol- lowing oral administration of Korean Red Ginseng extract treated with enzyme}

Physiological aspects of mouse dorsal skin surfaces after oral administration of KRGE were analyzed based on data obtained after 4 and 8 wk of UVB irradiation using an analytical system. The erythema index (EI) and water contents of the SC are shown in Fig. 4A and 4B, respectively. EI was significantly elevated by UVB exposure (up to 168\%) while dietary KRGE caused a decline in EI. Compared with the UVB control group, KRGE $0.06 \%$ (down to $22 \%$ ) was more efficient in decreasing EI than KRGE 0.24\% (down to 16\%). We also measured stratum corneum hydration (SCH). As shown in Fig. 4B, UVB suppressed SCH while both the KRGE $0.06 \%$ and KRGE $0.24 \%$ groups exhibited increased SCH by $133 \%$ and $111 \%$, respectively. Further, compared with the KRGE $0.24 \%$ group, the SCH levels of the KRGE 0.06\% group were much higher. 


\section{A}
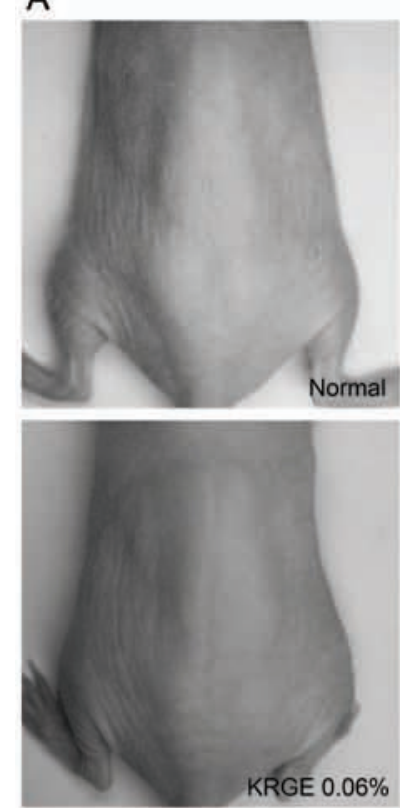

B
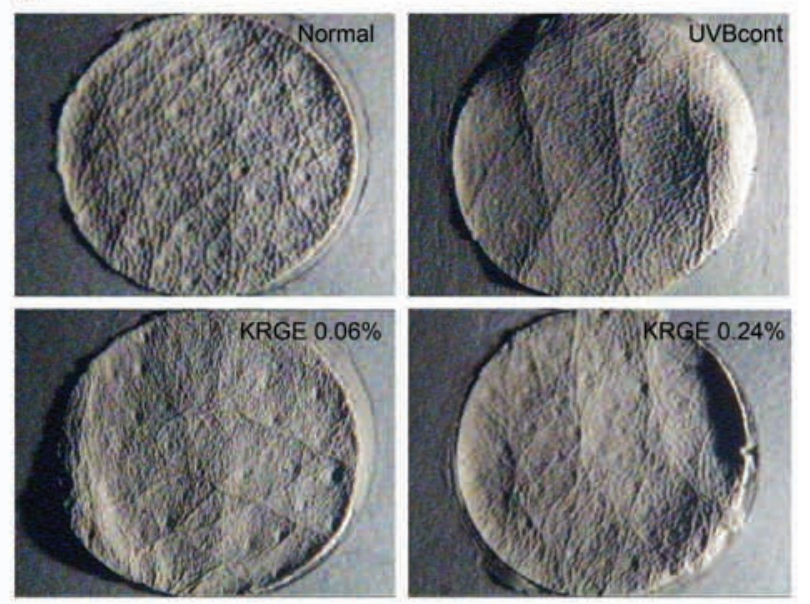

C

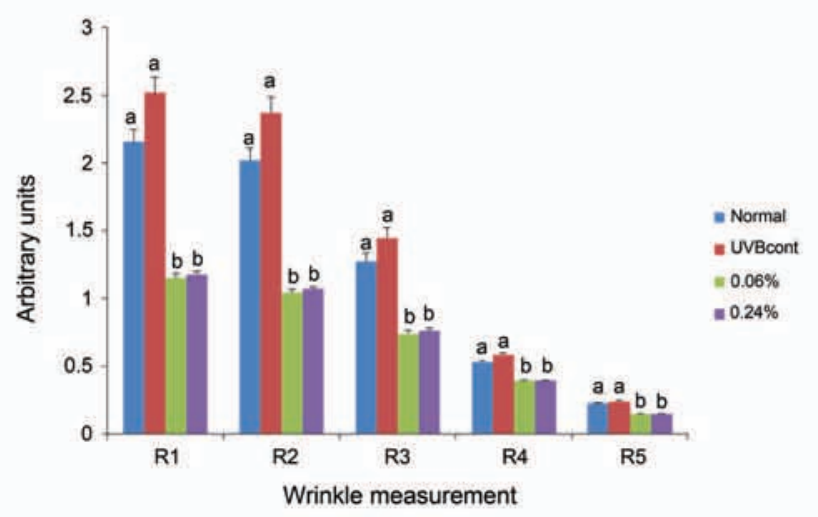

Fig. 2. Features of hairless mice dorsal skin (A), photographs of replicas $(B)$ and replica analysis $(C)$ taken from the dorsal skin of the mice. Normal (control diet only), UVBcont (UVB irradiation + control diet), Korean Red Ginseng extract treated with enzyme 0.06\% (UVB irradiation + diet containing $0.06 \% \mathrm{KRGE}$ ), and KRGE $0.24 \%$ (UVB irradiation + diet containing $0.24 \% \mathrm{KRGE}$ ). Values are mean \pm SD ( $n=5$ ). The data were evaluated for statistical significance with one-way ANOVA followed by Duncan's multiple range test. Means with the same letter are not significantly different. Thus, means with the different letter, e.g., 'a' or 'b' are statistically different.

Changes in MMP-1, procollagen type I, TGF- $\beta 1$, profilaggrin, and filaggrin expression following oral administration of KRGE

The mechanisms underlying the beneficial effects of KRGE were investigated at the level of protein expression. Specifically, we analyzed the expression of MMP1 , procollagen type I, and TGF- $\beta 1$. UVB irradiation decreased the abundance of procollagen type I and TGF- $\beta 1$ by $69 \%$ and $30 \%$, respectively, but dietary administration of KRGE reversed this effect in UVB irradiated mice. Furthermore, mice exposed to UVB and a diet contain- ing 0.06\% KRGE exhibited greatly increased expression of procollagen type I and TGF- $\beta 1$ protein expression (up to $479 \%$ and $175 \%$, respectively) compared with UVB control group (Fig. 5D). In the case of MMP-1, UVB increased protein expression by $169 \%$, while KRGE reversed this effect. Similar to procollagen type I and TGF$\beta 1$, the reversal of UVB-induced expression of MMP-1 was more prominent in the KRGE 0.06\% group than the KRGE 0.24\% group (Fig. 5A).

Profilaggrin and filaggrin were measured in both the epidermis and dermis by Western blot with $\alpha$-tubulin as 
A
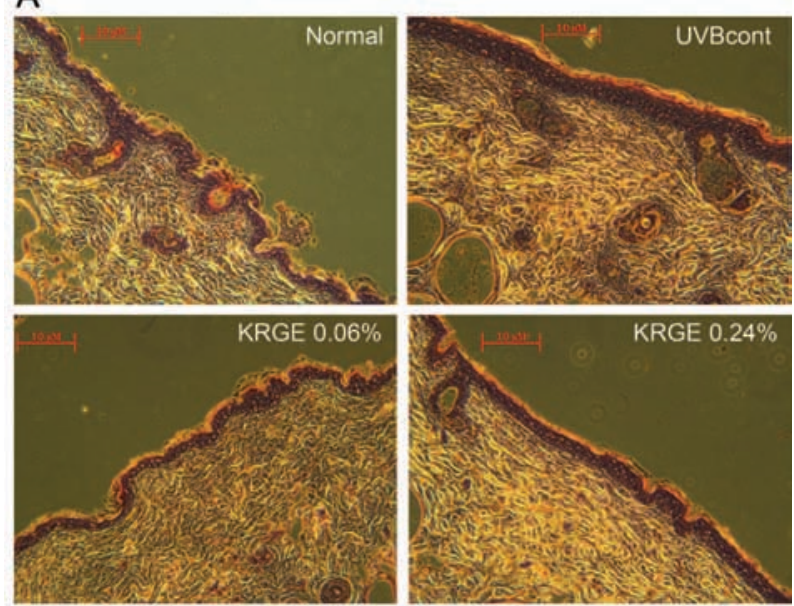

B
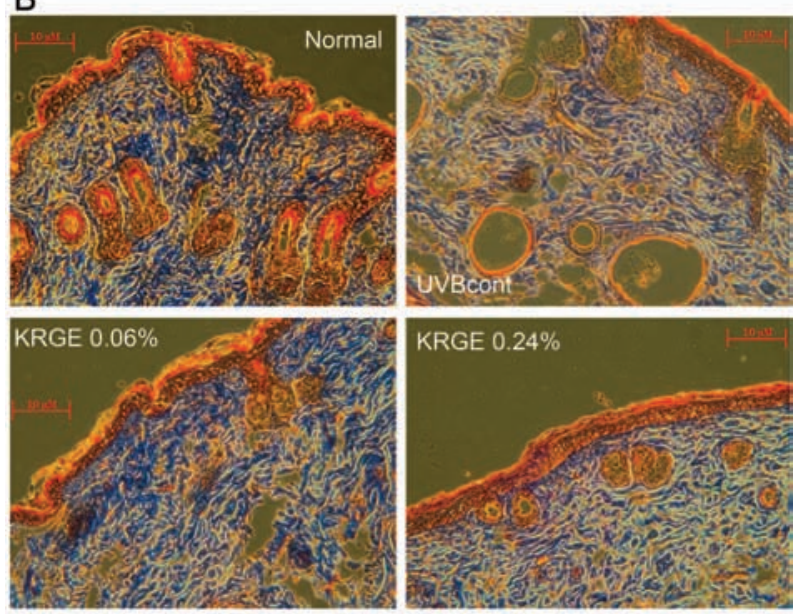

KRGE $0.24 \%$

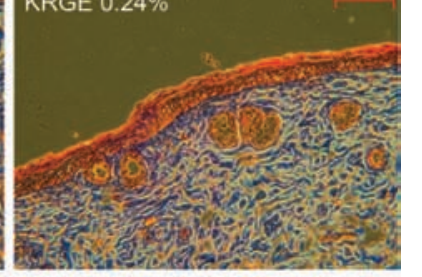

Fig. 3. Photomicrographs of H\&E-stained sections (A), and photomicrographs of Masson's trichrome-stained sections (B) from the dorsal skin of hairless mice. Normal (control diet only), UVBcont (UVB irradiation + control diet), Korean Red Ginseng extract treated with enzyme $0.06 \%$ (UVB irradiation + diet containing 0.06\% KRGE), and KRGE $0.24 \%$ (UVB irradiation + diet containing 0.24\% KRGE).

an internal reference. The expression of profilaggrin and filaggrin was clearly higher in the epidermis compared with the dermis. UVB exposure decreased expression of profilaggrin and filaggrin in both the epidermis and dermis, which was reversed by KRGE (Fig. 5B, C, respectively). Similar to the aforementioned effects of KRGE, the KRGE $0.06 \%$ group exhibited more significant changes than the KRGE $0.24 \%$ group (Fig. 5E, F).

\section{DISCUSSION}

MMPs play a primary role in the pathophysiological mechanism of photoaging [4]. Activated MMP-1, a member of the collagenase MMP subfamily, triggers
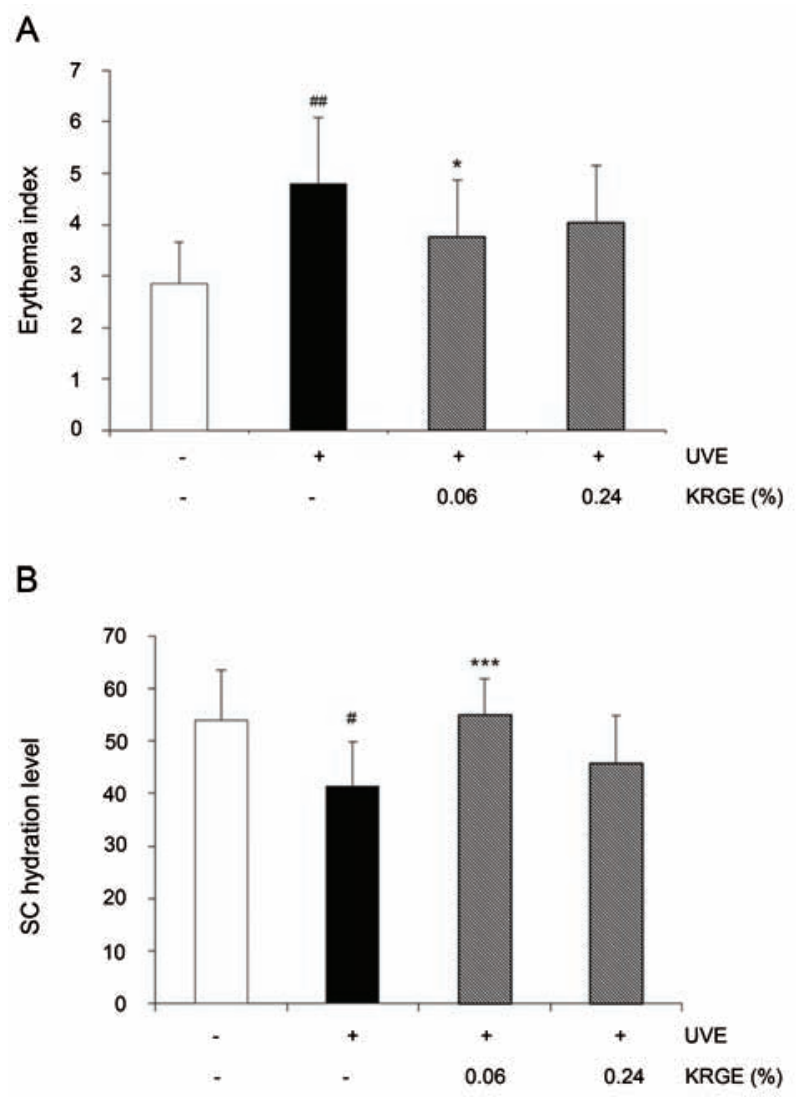

Fig. 4. Under the standardized room conditions of $22^{\circ} \mathrm{C}$ to $24^{\circ} \mathrm{C}$ and $55 \%$ to $60 \%$ humidity, erythema index (A) stratum corneum (SC) hydration $(B)$ of the hairless mice at the end of $10 \mathrm{wk}$. Values are means \pm SD. ${ }^{*}$ and * indicate significant differences $(p<0.05)$ between the UV (-) control and UV (+) control, respectively. ${ }^{\# \#} p<0.01$ vs. the normal control, ${ }^{\star} p<0.05$ and ${ }^{* * *} p<0.001$ vs. UVB-irradiated control. KRGE, Korean Red Ginseng extract treated with enzyme.

collagen degradation. Specifically, activated MMP-1 degrades collagen type I, a primary component of the extracellular matrix, which itself is a source of structural support in the dermis [24]. As reported previously, UV irradiation increases the expression of AP-1, which in turn interferes with the synthesis of collagen type I by blocking TGF- $\beta$ signaling $[25,26]$. In the present study, decreased expression of both TGF- $\beta 1$ and procollagen type I due to UVB irradiation was significantly restored when KRGE was administered. Specifically, KRGE markedly attenuated MMP-1 expression and increased expression of both TGF- $\beta 1$ and procollagen type I in skin tissue, with the KRGE $0.06 \%$ group exhibiting stronger effects than the KRGE $0.24 \%$ group. Given that a lower concentration of KRGE was more beneficial than higher doses, it will be important in future studies to determine an optimal dose of KRGE. Similarly, as we did not analyze the effect of KRGE on UVB-mediated changes in 
A

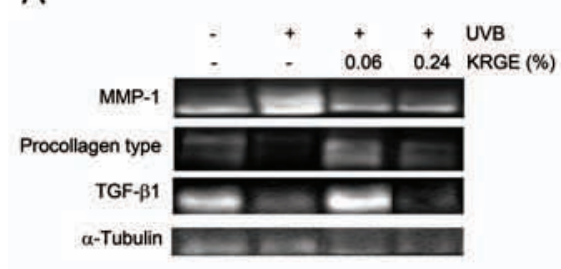

D

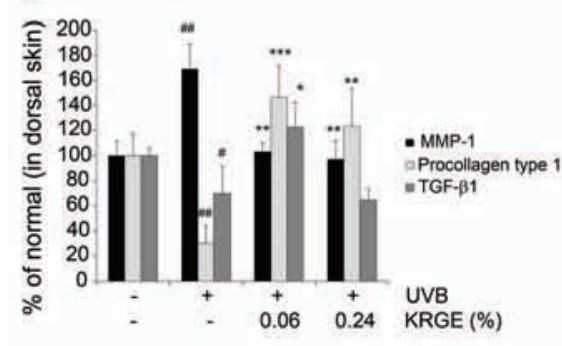

B

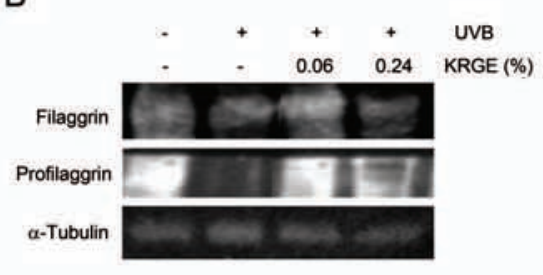

$\mathrm{E}$

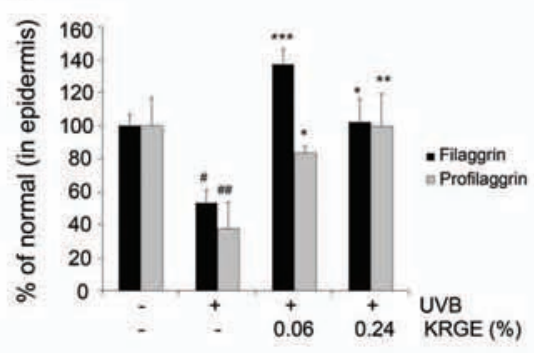

C

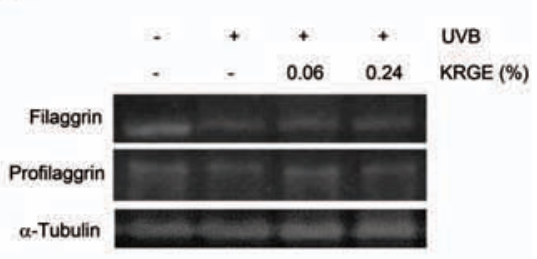

$\mathrm{F}$

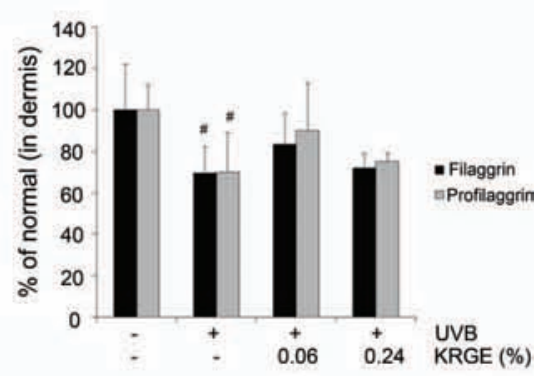

Fig. 5. The protein expressions of matrix-degrading metalloproteinase (MMP)-1, procollagen type I and transforming growth factor (TGF)- $\beta 1$ (A) in the dorsal skin; filaggrin and profilaggrin in epidermis (B) and in dermis (C) of hairless mice. The signal intensities from multiple experiments (D-F) were quantified, and the integrated areas were normalized, first to the corresponding value of $\alpha$-tubulin and then to the signal observed in the normal group. The bar graphs $(n=3)$ represent quantitative densitometric results of upper bands. Values are means \pm SD. ${ }^{*}$ and * indicate significant differences $(p<0.05)$ between the UV $(-)$ control and UV $(+)$ control, respectively. ${ }^{*} p<0.05$ and ${ }^{\#} p<0.01$ vs. the normal control, ${ }^{*} p<0.05,{ }^{* *} p<0.01$, and ${ }^{* \star} p<0.001$ vs. UVB-treated control. KRGE, Korean Red Ginseng extract treated with enzyme.

AP-1 expression, this should also be evaluated in future studies.

The SC is known as a barrier that protects underlying tissue from infection, dehydration, and exposure to chemical insults and mechanical stress. Within the SC, filaggrin monomers can become incorporated into lipid envelopes, which are responsible for the skin barrier function. In addition, filaggrin undergoes further processing in the upper SC to release free amino acids that assist in water retention [10,11]. In the present study, the results show that KRGE reversed the inhibitory effects of UVB irradiation on filaggrin and profilaggrin production in both the epidermis and dermis (Fig. 5B, C). Similarly, the results show that skin dryness induced by UVB exposure was reversed by KRGE, with the SC of the KRGE $0.06 \%$ group exhibiting increased moisture levels (Fig. 4B). Taken together, these results suggested that a low concentration of KRGE may play a role in restoring xerotic skin induced by UVB irradiation by increasing the production of both filaggrin and profilaggrin.

We previously investigated the protective effects of dietary KRGE extracts in UVB-irradiated skin aging in hairless mice, and observed up-regulation of collagen expression and down-regulation of MMP-1 secretion [23]. Likewise, Kim et al. [27] reported that dietary red ginseng stimulates epidermal hydration and ceramide production in UV-irradiated hairless mice. In the present study, we found that KRGE positively regulated not only collagen and MMP-1 production, but also epidermal hydration and filaggrin. Although there have been numerous studies conducted on the effects of red ginseng on skin anti-aging $[23,28,29]$, to the best of our knowledge there have been no studies evaluating the effects of low dose dietary KRGE on skin photoaging and hydration. The major ginsenosides, including Rb1, Rb2, and Rc, as glycosylated ginsenosides are poorly absorbed into the gastrointestinal tract [30]. In contrast, the absorption of the minor ginsenosides as deglycosylated ginsenosides is quite easy in the bloodstream [31]. Enzymatic transformation technique may be responsible for high absorption of the ginsenosides by transforming the major ginsenosides to the minor ginsenosides via the selective hydrolysis of the sugar moieties of ginsenosides. These may lead that a low dose of KRGE had stronger protective effects against UVB-induced photodamages than a high dose of KRGE.

According to the results of the present study, there was no difference with respect to reduction of visible wrinkle formation between low (0.06\%) and high doses $(0.24 \%)$ dietary KRGE. However, with respect to histologic, physiologic, and molecular changes in skin tissue, the protective effects of KRGE with a low dose of $0.06 \%$ dietary KRGE were more significant than that of high dose KRGE (0.24\%). In conclusion, a low dose of KRGE as 
a dietary supplement has potential beneficial effects on photoaging. Furthermore, it is likely that KRGE may have useful effects in both bio-health skin care products and cosmetics.

\section{ACKNOWLEDGEMENTS}

This research was supported by Korea Institute of Planning and Evaluation for Technology in Food, Agriculture, Forestry and Fisheries (iPET, 810006-03-1SB120), Republic of Korea.

\section{REFERENCES}

1. Farage MA, Miller KW, Elsner P, Maibach HI. Functional and physiological characteristics of the aging skin. Aging Clin Exp Res 2008;20:195-200.

2. Miyauchi H, Horio T, Asada Y. The effect of ultraviolet radiation on the water-reservoir functions of the stratum corneum. Photodermatol Photoimmunol Photomed 19921993;9:193-197.

3. Tanaka H, Okada T, Konishi H, Tsuji T. The effect of reactive oxygen species on the biosynthesis of collagen and glycosaminoglycans in cultured human dermal fibroblasts. Arch Dermatol Res 1993;285:352-355.

4. Fisher GJ, Kang S, Varani J, Bata-Csorgo Z, Wan Y, Datta S, Voorhees JJ. Mechanisms of photoaging and chronological skin aging. Arch Dermatol 2002;138:1462-1470.

5. Park HM, Hwang E, Lee KG, Han SM, Cho Y, Kim SY. Royal jelly protects against ultraviolet B-induced photoaging in human skin fibroblasts via enhancing collagen production. J Med Food 2011;14:899-906.

6. Fisher GJ, Datta SC, Talwar HS, Wang ZQ, Varani J, Kang S, Voorhees JJ. Molecular basis of sun-induced premature skin ageing and retinoid antagonism. Nature 1996;379:335-339.

7. Fisher GJ, Quan T, Purohit T, Shao Y, Cho MK, He T, Varani J, Kang S, Voorhees JJ. Collagen fragmentation promotes oxidative stress and elevates matrix metalloproteinase-1 in fibroblasts in aged human skin. Am J Pathol 2009;174:101-114.

8. Pearse AD, Gaskell SA, Marks R. Epidermal changes in human skin following irradiation with either UVB or UVA. J Invest Dermatol 1987;88:83-87.

9. Ovaere P, Lippens S, Vandenabeele P, Declercq W. The emerging roles of serine protease cascades in the epidermis. Trends Biochem Sci 2009;34:453-463.

10. Kambayashi H, Yamashita M, Odake Y, Takada K, Funasaka Y, Ichihashi M. Epidermal changes caused by chronic low-dose UV irradiation induce wrinkle formation in hairless mouse. J Dermatol Sci 2001;27 Suppl 1:S19-S25.

11. Biniek K, Levi K, Dauskardt RH. Solar UV radiation reduces the barrier function of human skin. Proc Natl Acad Sci U S A 2012;109:17111-17116.

12. Park HJ, Kim DH, Park SJ, Kim JM, Ryu JH. Ginseng in traditional herbal prescriptions. J Ginseng Res 2012;36:225-241.

13. Park CS, Yoo MH, Noh KH, Oh DK. Biotransformation of ginsenosides by hydrolyzing the sugar moieties of ginsenosides using microbial glycosidases. Appl Microbiol Biotechnol 2010;87:9-19.

14. Bae HM, Kim SS, Cho CW, Yang DC, Ko SK, Kim KT. Antioxidant activities of ginseng seeds treated by autoclaving. J Ginseng Res 2012;36:411-417.

15. Kim CS, JO YJ, Park SH, Kim HJ, Han JY, Hong JT, Cheong JH, Oh KW. Anti-stress effects of ginsenoside Rg3-standardized ginseng extract in restraint stressed animals. Biomol Ther 2010;18:219-225.

16. Lee MJ, Kim EH, Rhee DK. Effects of Panax ginseng on stress. J Ginseng Res 2008;32:8-14.

17. Quan FS, Compans RW, Cho YK, Kang SM. Ginseng and Salviae herbs play a role as immune activators and modulate immune responses during influenza virus infection. Vaccine 2007;25:272-282.

18. Bensky D, Clavey S, Stoger E, Gamble A. Chinese herbal medicine: materia medica. 3rd ed. Seattle: Eastland Press, 2004.

19. Kim HD, Ha SE, Kang JR, Park JK. Effect of Korean red ginseng extract on cell death responses in peroxynitritetreated keratinocytes. J Ginseng Res 2010;34:205-211.

20. Hong CE, Lyu SY. Anti-inflammatory and anti-oxidative effects of Korean red ginseng extract in human keratinocytes. Immune Netw 2011;11:42-49.

21. Lee H, Lee JY, Song KC, Kim J, Park JH, Chun KH, Hwang GS. Protective effect of processed Panax ginseng, sun ginseng on UVB-irradiated human skin keratinocyte and human dermal fibroblast. J Ginseng Res 2012;36:6877.

22. Yue CJ, Zhou X, Zhong JJ. Protopanaxadiol 6-hydroxylase and its role in regulating the ginsenoside heterogeneity in Panax notoginseng cells. Biotechnol Bioeng 2008;100:933-940.

23. Kang TH, Park HM, Kim YB, Kim H, Kim N, Do JH, Kang C, Cho Y, Kim SY. Effects of red ginseng extract on UVB irradiation-induced skin aging in hairless mice. J Ethnopharmacol 2009;123:446-451.

24. Ichihashi M, Ueda M, Budiyanto A, Bito T, Oka M, Fukunaga M, Tsuru K, Horikawa T. UV-induced skin damage. Toxicology 2003;189:21-39.

25. Varani J, Spearman D, Perone P, Fligiel SE, Datta SC, 
Wang ZQ, Shao Y, Kang S, Fisher GJ, Voorhees JJ. Inhibition of type I procollagen synthesis by damaged collagen in photoaged skin and by collagenase-degraded collagen in vitro. Am J Pathol 2001;158:931-942.

26. Pittelkow MR, Coffey RJ Jr, Moses HJ. Keratinocytes produce and are regulated by transforming growth factors. Ann N Y Acad Sci 1988;548:211-224.

27. Kim H, Oh I, Park KH, Kim NM, Do JH, Cho Y. Stimulatory effect of dietary red ginseng on epidermal hydration and ceramide levels in ultraviolet-irradiated hairless mice. J Med Food 2009;12:746-754.

28. Chen JK, Chen TT. Chinese medical herbology and pharmacology. City of Industry: Art of Medicines Press, 2004.

29. Kim YG, Sumiyoshi M, Kawahira K, Sakanaka M,
Kimura Y. Effects of red ginseng extract on ultraviolet B-irradiated skin change in C57BL mice. Phytother Res 2008;22:1423-1427.

30. Tawab MA, Bahr U, Karas M, Wurglics M, Schubert-Zsilavecz M. Degradation of ginsenosides in humans after oral administration. Drug Metab Dispos 2003;31:10651071.

31. Karikura M, Miyase T, Tanizawa H, Taniyama T, Takino Y. Studies on absorption, distribution, excretion and metabolism of ginseng saponins. VII. Comparison of the decomposition modes of ginsenoside-Rb1 and -Rb2 in the digestive tract of rats. Chem Pharm Bull (Tokyo) 1991;39:2357-2361. 